\title{
Eosinophilic pneumonia in a traveller returning from Mexico
}

\author{
Jan Hajek MD FRCPC ${ }^{1}$, Subhash K Mohan MLT ART ${ }^{2}$, Theodore K Marras MD FRCPC ${ }^{3}$
}

\section{CASE PRESENTATION}

A previously healthy 45-year-old Korean-born man presented with pleuritic chest pain and a dry cough in February 2005. His symptoms began one week after returning home to Toronto, Ontario, following a trip to northern Mexico, where he had spent several days in a large city attending meetings. He denied having fever, rash or arthralgia. His chest pain and cough were initially managed symptomatically with an analgesic and a cough supressant. However, the symptoms persisted and after one week, he was referred to a respirologist for further investigation and management. His physical examination was normal. His complete blood count revealed marked eosinophilia $\left(6.0 \times 10^{9} / \mathrm{L}\right)$ and his chest radiograph documented a right lung infiltrate. A computed tomography scan confirmed consolidation in the right upper lobe and revealed centrilobular nodules in a tree-in-bud pattern, right hilar and mediastinal lymphadenopathy and small nodules (less than $0.5 \mathrm{~mm}$ ) scattered throughout both lungs.

He underwent bronchoscopic alveolar lavage and transbronchial biopsy. Cytology of the bronchoscopic alveolar lavage fluid revealed marked eosinophilia. Gram stain and calcofluor white (fungal) stain were negative. Histopathology of the biopsy documented nonspecific eosinophilic bronchitis. There were no identifiable organisms with hematoxylin and eosin, periodic acid-Schiff or GrocottGomori methenamine-silver stains of the tissue. A microbiological test was performed.

What was the diagnosis and how was it made?

\footnotetext{
${ }^{1}$ Division of Infectious Diseases, University of Toronto; ${ }^{2}$ Department of Microbiology, Toronto Medical Laboratories and Mount Sinai Hospital; ${ }^{3}$ Division of Respirology, Toronto Western Hospital, University of Toronto, Toronto, Ontario

Correspondence: Dr Jan Hajek, North York General Hospital, 4001 Leslie Street, GW-33, Toronto, Ontario M2K 1E1. Telephone 416-756-6130, fax 416-756-6449, e-mail jan.hajek@utoronto.ca

Received and accepted for publication July 19, 2007
} 


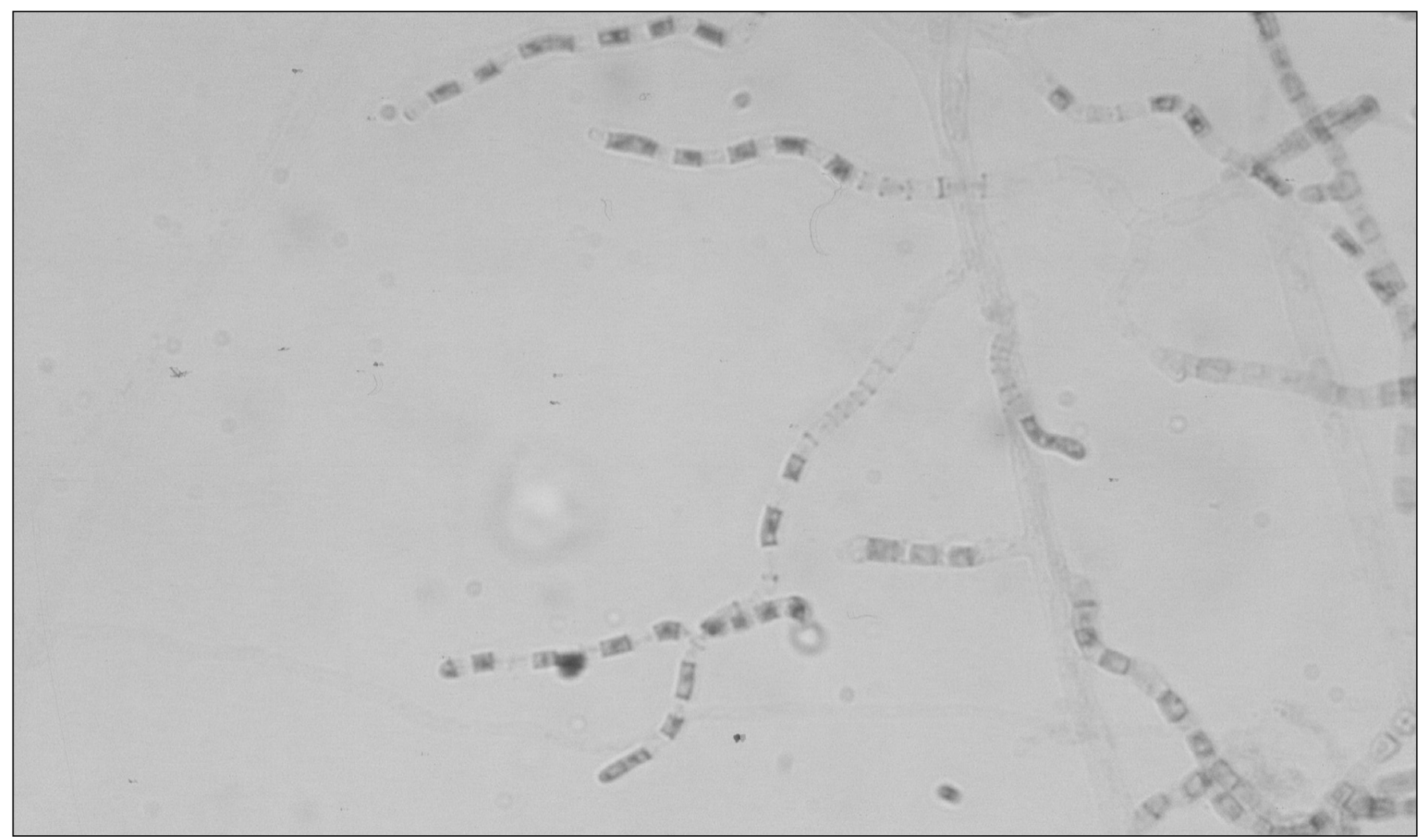

Figure 1) Lactophenol aniline blue wet mount of fungal culture using a Scotch tape preparation and examined microscopically at $\times 400$ magnification. Rectangular and barrel-shaped alternating arthroconidia, characteristic of the Coccidioides species, are seen

\section{DIAGNOSIS}

After five days of incubation, cultures on blood agar plates grew a white mould. Microscopic examination of a lactophenol aniline blue preparation revealed 'barrel-shaped' mycelial arthroconidia, characteristic of the highly pathogenic fungus Coccidioides (Figure 1). The samples were forwarded to a reference laboratory and molecular testing confirmed the presence of Coccidioides species.

The diagnosis of coccidioidomycosis was made three weeks after symptom onset, by which time the patient's pleuritic chest pain had resolved and his chest $\mathrm{x}$-ray had improved. Coccidioides serology, by the complement fixation test, was positive at a titre of 1:4. Given the patient's spontaneous clinical resolution, the decision was made to withhold antifungal agents.

At follow-up six months later, he remained asymptomatic. The chest radiograph changes and eosinophilia had resolved; his serology remained unchanged at a titre of 1:4.

\section{DISCUSSION}

Coccidioidomycosis is an invasive fungal infection caused by the inhalation of the arthroconidia (spores) of the dimorphic fungus Coccidioides. Although Coccidioides species (Coccidioides immitis and Coccidioides posadasii) are found in a geographically restricted habitat, infection can manifest as illness in returning travellers and is increasingly diagnosed in nonendemic areas (1). Although intense exposure to dust or soil is a risk factor, most infections occur without such an exposure history.

C immitis is endemic to regions in the southwestern United States - common destinations for Canadian snowbirds.
C posadasii is more widespread and found in regions of Mexico and South and Central America with similar climate and soil conditions. The two species have otherwise indistinguishable clinical features (1).

Following inhalation, the tiny arthroconidia $(3 \mu \mathrm{m}$ to $5 \mu \mathrm{m})$ can travel to the alveoli, where they undergo a dimorphic shift and develop into large spherules $(40 \mu \mathrm{m}$ to $100 \mu \mathrm{m})$ filled with endospores. These spherules incite a granulomatous reaction and a strong Th1-type, cell-mediated immune response is required to control the infection. Th2-type immune responses may confer susceptibility to dissemination. Peripheral eosinophilia, associated with Th2 responses, has been suggested to be a marker of disseminated disease (2). However, eosinophilia may simply represent a hypersensitivity reaction (3).

The majority of individuals infected with Coccidioides infection remain asymptomatic (4). Fewer than 50\% develop clinical illness, most commonly a respiratory illness, mimicking typical community-acquired pneumonia. Approximately 50\% have associated peripheral eosinophilia and 25\% develop erythema nodosum which are useful diagnostic clues (5).

The primary illness is typically self-limited. Fewer than $5 \%$ of individuals develop progressive or persistent pulmonary disease, and fewer than 1\% develop extrapulmonary complications. However, the risk of disseminated disease is high, up to $50 \%$, in immunocompromised patients. Typical sites of dissemination include the skin, bones and the meninges; however, any organ can be involved. Disseminated disease may coincide with symptoms of primary pulmonary infection or may occur weeks to months later. 
Coccidioides spherules can persist within granulomas and can disseminate in the setting of future immunosuppression following a period of latency (6). Of note, approximately 50\% of Coccidioides infections in patients with AIDS are diagnosed outside of endemic regions (7).

Laboratory diagnosis of Coccidioides can be made by the direct visualization of the characteristic arthroconidia or spherules in sputum samples or tissue biopsies. However, direct visualization is only $40 \%$ sensitive compared with culture (8). Coccidioides grows readily on most media within five days. The definitive identification of Coccidioides from culture typically requires the use of exoantigen tests or DNA probes.

Due to its virulence and potential for aerosol, the microbiology laboratory should be advised of the possibility of Coccidioides so that proper precautions can be taken (biosafety level 3 practices).

\section{REFERENCES}

1. Galgiani JN. Coccidioides species. In: Mandell GM, Bennet JE, Dolin R, eds. Principles and Practice of Infectious Diseases, 6th edn. Philadelphia: Elsevier Inc, 2005:3040-51.

2. Harley WB, Blaser MJ. Disseminated coccidioidomycosis associated with extreme eosinophilia. Clin Infect Dis 1994;18:627-9.

3. Lombard CM, Tazelaar HD, Krasne DL. Pulmonary eosinophilia in coccidioidal infections. Chest 1987;91:734-6.

4. Stevens DA. Coccidioidomycosis. N Engl J Med 1996;332:1077-82.

5. Feldman BS, Snyder LS. Primary pulmonary coccidioidomycosis. Semin Respir Infect 2001;16:231-7.

6. Blair JE, Logan JL. Coccidioidomycosis in solid organ transplantation. Clin Infect Dis 2001;33:1536-44.
Diagnosis is frequently made by serology. Detection of complement-fixing antibody has good specificity, and high titres (greater than 1:16) are markers for increased risk of complications (9).

Updated treatment guidelines for coccidioidomycosis have recently been published (10). In general, given the wide spectrum of disease, treatment is individualized according to the extent of infection and risk factors for dissemination. Although recommendations vary from observation alone for uncomplicated pneumonia to life-long systemic antifungals and consideration of intrathecal amphotericin B for meningitis, all patients require clinical follow-up after primary infection.

The present case highlights the need to remember this re-emerging fungal pathogen as a cause of eosinophilic pneumonia and an important cause of illness in returning travellers.

7. Jones JL, Fleming PL, Ciesielski CA, Hu DJ, Kaplan JE, Ward JW. Coccidioidomycosis among persons with AIDS in the United States. J Infect Dis 1995;171:961-6.

8. DiTomasso JP, Ampel NM, Sobonya RE, Bloom JW.

Bronchoscopic diagnosis of pulmonary coccidioidomycosis. Comparison of cytology, culture, and transbronchial biopsy. Diagn Microbiol Infect Dis 1994;18:83-7.

9. Crum NF, Lederman ER, Stafford CM, Parrish JS, Wallace MR. Coccidioidomycosis: A descriptive survey of a reemerging disease. Clinical characteristics and current controversies. Medicine (Baltimore) 2004;83:149-75

10. Galgiani JN, Ampel NM, Blair JE, et al; Infectious Diseases Society of America. Coccidioidomycosis. Clin Infect Dis 2005;41:1217-23. 


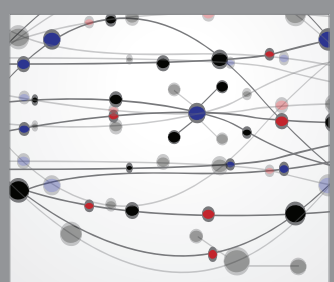

The Scientific World Journal
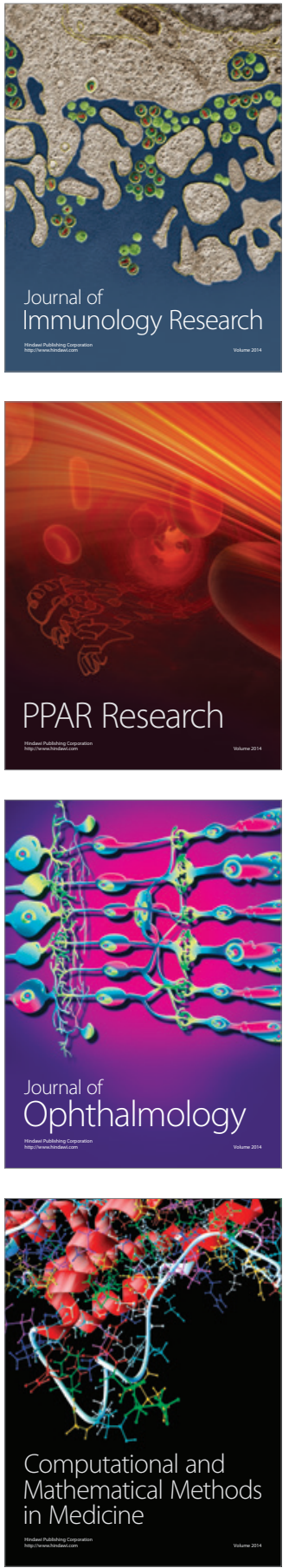

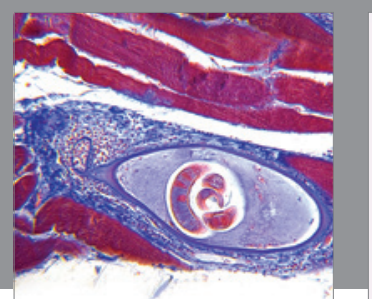

Gastroenterology Research and Practice

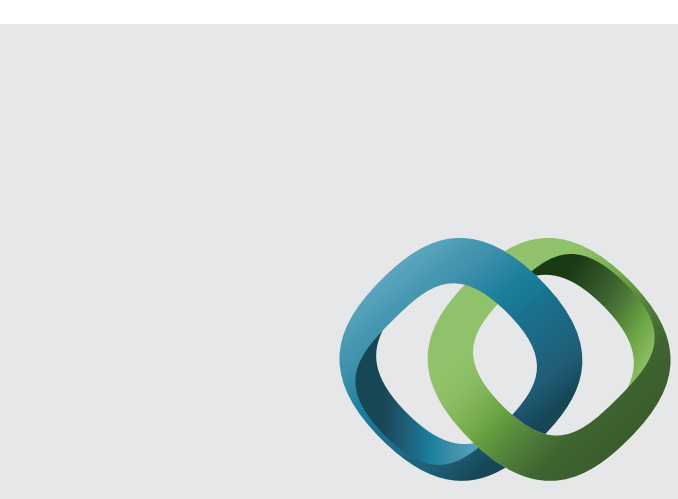

\section{Hindawi}

Submit your manuscripts at

http://www.hindawi.com
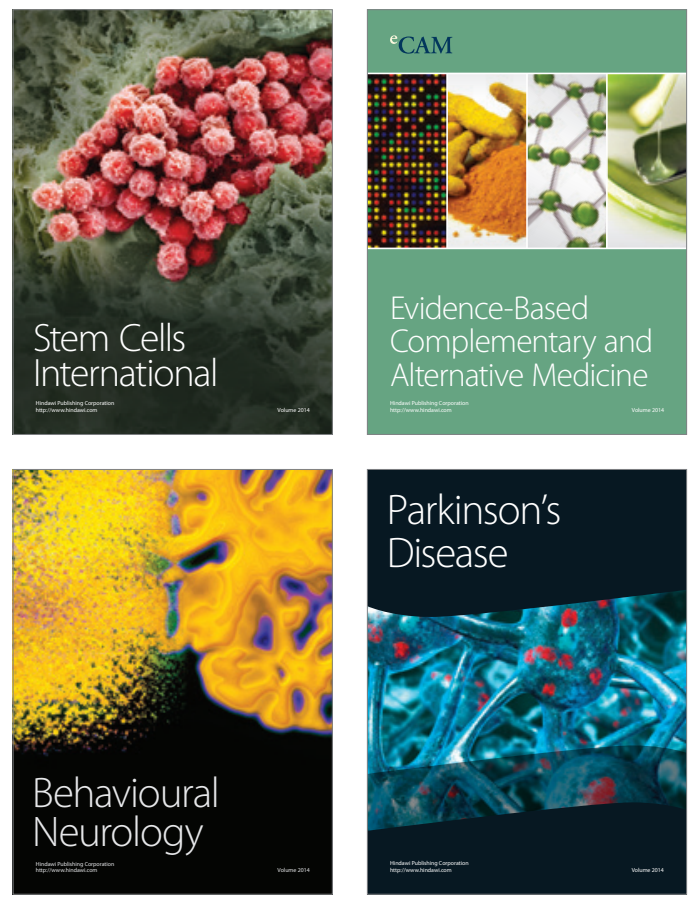
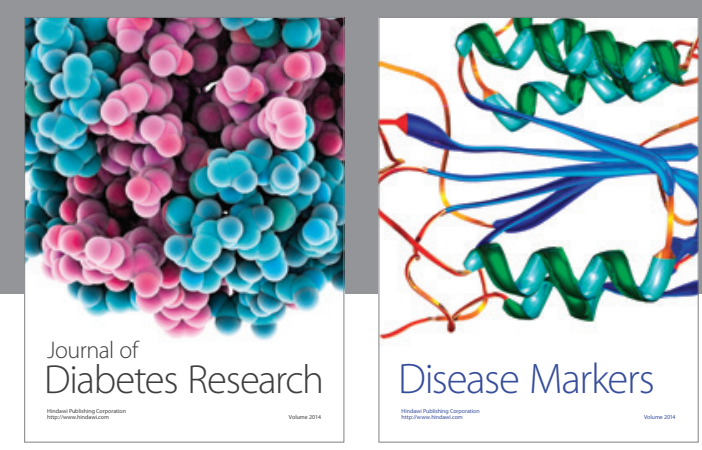

Disease Markers
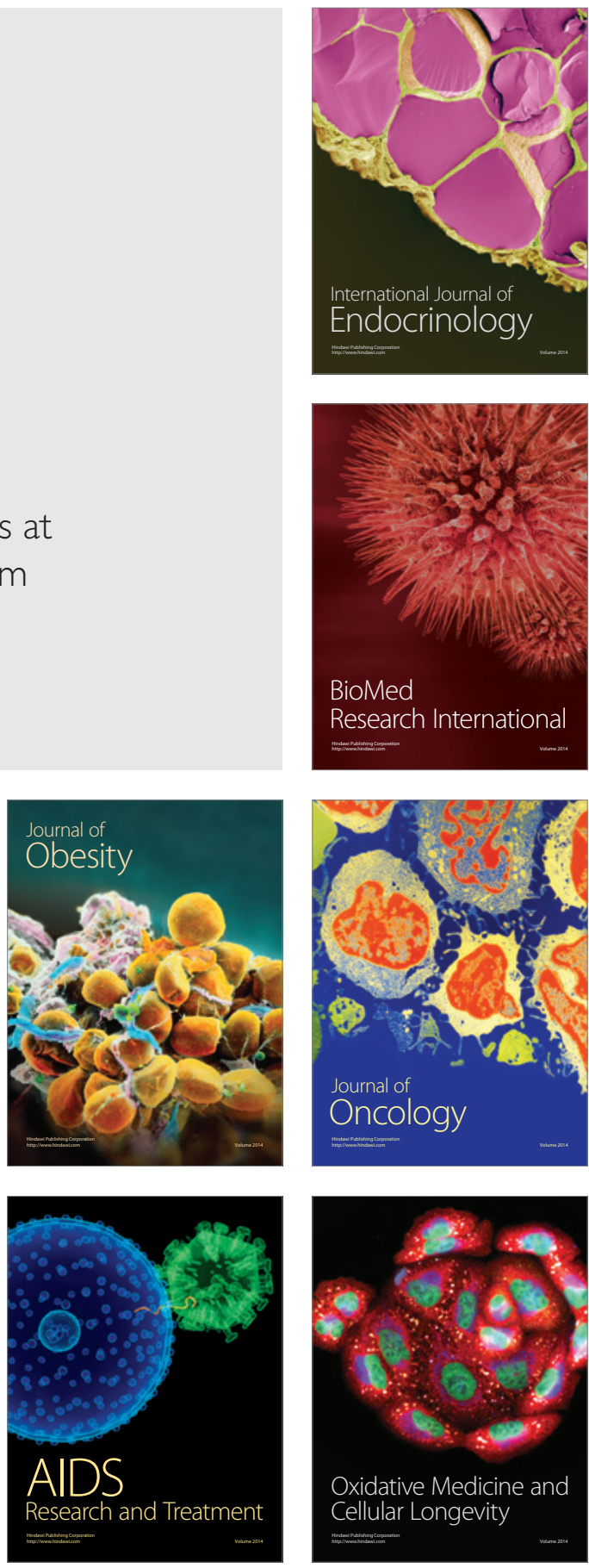